\title{
Integer Factorization with a Neuromorphic Sieve
}

\author{
John V. Monaco and Manuel M. Vindiola \\ U.S. Army Research Laboratory \\ Aberdeen Proving Ground, MD 21005 \\ Email: john.v.monaco2.civ@mail.mil,manuel.m.vindiola.civ@mail.mil
}

\begin{abstract}
The bound to factor large integers is dominated by the computational effort to discover numbers that are smooth, typically performed by sieving a polynomial sequence. On a von Neumann architecture, sieving has log-log amortized time complexity to check each value for smoothness. This work presents a neuromorphic sieve that achieves a constant time check for smoothness by exploiting two characteristic properties of neuromorphic architectures: constant time synaptic integration and massively parallel computation. The approach is validated by modifying msieve, one of the fastest publicly available integer factorization implementations, to use the IBM Neurosynaptic System (NS1e) as a coprocessor for the sieving stage.
\end{abstract}

\section{INTRODUCTION}

A number is said to be smooth if it is an integer composed entirely of small prime factors. Smooth numbers play a critical role in many interesting number theoretic and cryptography problems, such as integer factorization [6]. The presumed difficulty of factoring large composite integers relies on the difficultly of discovering many smooth numbers in a polynomial sequence, typically performed through a process called sieving. The detection and generation of smooth numbers remains an ongoing multidisciplinary area of research which has seen both algorithmic and implementation advances in recent years [2].

This work demonstrates how current and near future neuromorphic architectures can be used to efficiently detect smooth numbers in a polynomial sequence. The neuromorphic sieve exploits two characteristic properties of neuromorphic architectures to achieve asymptotically lower bounds on smooth number detection, namely constant time synaptic integration and massively parallel computation. Sieving is performed by a population of leaky integrate-and-fire (LIF) neurons whose dynamics are simple enough to be implemented on a range of current and future architectures. Unlike the traditional CPUbased sieve, the factor base is represented in space (as spiking neurons) and the sieving interval in time (as successive time steps). Integer factorization is achieved using a neuromorphic coprocessor for the sieving stage, alongside a CPU.

\section{INTEGER FACTORIZATION}

Integer factorization is presumed to be a difficult task when the number to be factored is the product of two large primes. Such a number $n=p q$ is said to be a semiprime ${ }^{1}$ for primes $p$ and $q, p \neq q$. As $\log _{2} n$ grows, i.e., the number of bits

\footnotetext{
${ }^{1}$ Not to be confused with pseudoprime, which is a probable prime.
}

to represent $n$, the computational effort to factor $n$ by trial division grows exponentially.

Dixon's factorization method attempts to construct a congruence of squares, $x^{2} \equiv y^{2} \bmod n$. If such a congruence is found, and $x \not \equiv \pm y \bmod n$, then $\operatorname{gcd}(x-y, n)$ must be a nontrivial factor of $n$. A class of subexponential factoring algorithms, including the quadratic sieve, build on Dixon's method by specifying how to construct the congruence of squares through a linear combination of smooth numbers [5].

Given smoothness bound $B$, a number is $B$-smooth if it does not contain any prime factors greater than $B$. Additionally, let $\mathbf{v}=\left[e_{1}, e_{2}, \ldots, e_{\pi(B)}\right]$ be the exponents vector of a smooth number $s$, where $s=\prod_{1 \leq i \leq \pi(B)} p_{i}^{v_{i}}, p_{i}$ is the $i$ th prime, and $\pi(B)$ is the number of primes not greater than $B$. With a set of $\pi(B)+1$ unique smooth numbers $S=\left\{s_{1}, s_{2}, \ldots, s_{\pi(B)+1}\right\}$, a perfect square can be formed through some linear combination of the elements of $S, y^{2}=\prod_{s_{i} \in S} s_{i}$. The reason for this is that there exists at least one linear dependency among a subset of the $\pi(B)+1$ exponents vectors that contain $\pi(B)$ elements each. Gaussian elimination or block Lanczos algorithm can be used to uncover this linear dependency [4].

Smooth numbers are detected by sieving a polynomial sequence. Sieving relies on the fact that for each prime $p$, if $p \mid f(x)$ then $p \mid f(x+i p)$ for any integer $i$ and polynomial $f$. To sieve the values $f(x)$, for $0 \leq x<M$, on a von Neumann architecture, a length $M$ array is initialized to all zeros. For each polynomial root $r$ of each prime $p$ in the factor base, $\ln p$ is added to array locations $r+i p$ for $i=0,1, \ldots, \frac{M}{p}$. This step can be performed using low precision arithmetic, such as with integer approximations to $\ln p$. After looping through each prime in the factor base, array values above a certain threshold will correspond to polynomial values that are smooth with high probability ${ }^{2}$. This process is referred to hereafter as CPU-based sieving.

Due to errors resulting from low precision arithmetic, some of the array values above the threshold will end up being not smooth and some values that are smooth will remain below the threshold. The threshold controls a tradeoff between the false positive rate (FPR) and false negative rate (FNR), from which a receiver operating characteristic (ROC) curve is obtained. Since the actual factorizations are lost after sieving, the smooth candidates must subsequently be factored over $\mathcal{F}$, which also serves as a definite check for smoothness. Factoring a number with small prime factors can be done efficiently, and this effort

\footnotetext{
${ }^{2}$ By exploiting the fact that $\ln a b=\ln a+\ln b$.
} 
can be neglected as long as there are not too many false positives [1].

The quadratic sieve [5] detects smooth numbers of the form

$$
f(x)=(x+\lceil\sqrt{n}\rceil)^{2}-n
$$

where $x=-\frac{M}{2}, \ldots, \frac{M}{2}-1$. The factor base $\mathcal{F}$ contains primes $p$ up to $B$ such that $n$ is a quadratic residue modulo $p$, i.e., $r^{2} \equiv n \bmod p$ for some integer $r$. This ensures that each prime in the factor base (with the exception of 2) has two modular roots to the equation $f(x) \equiv 0 \bmod p$, increasing the probability that $p \mid f(x)$. If $\mathcal{F}$ contains $b$ primes, then at least $b+1$ smooth numbers are needed to form the congruence of squares.

It is the sieving stage of the quadratic sieve that is the focus of this work. Sieving comprises the bulk of the computational effort in the quadratic sieve and the relatively more complex number field sieve (NFS) [1]. On a von Neumann architecture, sieving requires at least $\frac{1}{2} M+M \sum_{p \in \mathcal{F} \backslash 2} \frac{2}{p}$ memory updates where $\mathcal{F} \backslash 2$ is the set of factor base primes excluding 2 . Given likelihood $u^{-u}$ of any single polynomial value being smooth, where $u=\frac{1}{2} \frac{\ln n}{\ln B}$, an optimal choice of $B$ is $\exp \left(\frac{1}{2} \sqrt{\ln n \ln \ln n}\right)$ [1]. This yields a total runtime of $B^{2}$, where the amortized time to sieve each value in the interval is $\ln \ln B$.

\section{NeUROMORPHIC SIEVE}

The neuromorphic sieve represents the factor base in space, as tonic spiking neurons, and the sieving interval in time through a one-to-one correspondence between time and the polynomial sequence. Let $t \equiv\left(x-x_{\text {min }}\right)$, where $t$ is time, $x$ is the sieving location that corresponds to $t$, and $x_{\text {min }}$ is the first sieving value. Then polynomial values can be calculated by $f(x)=f\left(t+x_{m i n}\right)$. Each tonic neuron corresponds to a prime (or a power of a prime) in the factor base and spikes only when it divides the current polynomial value. If enough neurons spike at time $t$, then $f(x)$ is likely smooth since each neuron represents a factor of $f(x)$. This formulation reverses the roles of space and time from the CPU-based sieve, in which the sieving interval is represented in space, as an array, and a doubly nested loop iterates over over primes in the factor base and locations in the sieving interval.

Construction of the neuromorphic sieve is demonstrated through an example using the semiprime $n=91$, quadratic polynomial $f(x)=(x+\lceil\sqrt{91}\rceil)^{2}-91$, and sieving interval $x=-5, \ldots, 4$. The smoothness bound is set to $B=5$. This creates a prime factor base $\mathcal{F}=\{2,3,5\}$, the primes up to $B$ such that the Legendre symbol $\left(\frac{n}{p}\right)=1$, i.e., $n$ is a quadratic residue modulo each of the primes in the factor base. Sieving is also performed with prime powers, $p^{e}$ for $e>1$, that do not exceed the magnitude of the polynomial, namely $3^{2}, 3^{3}$, and $5^{2}$. Powers of 2 are not included since they do not have any modular roots to the equation $f(x) \equiv 0 \bmod 2^{e}$ for $e>1$.

To achieve a constant-time check for smoothness, a spiking neural network is composed of three layers that form a tree

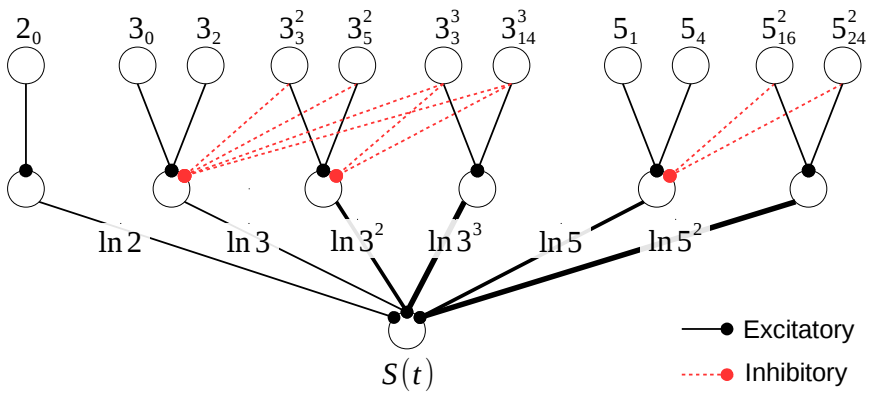

Fig. 1: Example neuromorphic sieve network. Top layer contains tonic spiking neurons; middle layer selects the highest prime power; bottom layer performs the test for smoothness.

structure (Figure 11). The top layer contains tonic spiking neurons for each prime in the factor base as well as prime powers, up to the magnitude of the polynomial. The middle layer selects the highest prime power that divides the polynomial value. The bottom layer contains a single neuron that performs a test for smoothness by integrating the log-weighted factors. The middle and bottom layers are stateless, while the dynamics in the top layer encode successive polynomial values.

For each modular root $r$ of each prime power $p^{e}$ (including factor base primes, for which $e=1$ ), designate a neuron that will spike with period $p^{e}$ and phase $r$ (Figure 11, top layer, given by $p_{r}^{e}$, where subscripts denote the modular root). Due to the equivalence between $t$ and $f(x)$, this neuron will spike only when $p^{e} \mid f(x)$. It is also the case that if $p^{e} \mid f(x)$ then $p^{e} \mid f\left(x+i p^{e}\right)$ for any integer $i$, thus only tonic spiking behavior for each modular root is required.

The tonic spiking neurons are connected through excitatory synapses to a postsynaptic neuron that spikes if either modular root spikes, i.e., if $p^{e} \mid f(x)$ for modular roots $r_{1}$ and $r_{2}$ of prime $p^{e}$ (Figure 1. middle layer). Using a LIF neuron model, this behavior is achieved using synapse weights of the same magnitude as the postsynaptic neuron threshold. Inhibitory connections are formed between the prime powers in the top layer and lower prime powers in the middle layer so that higher powers suppress the spiking activity of the lower powers. This ensures that only the highest prime power that divides the current polynomial value is integrated by the smoothness neuron (Figure 1, bottom layer). Synapse connections to the smoothness neuron are weighted proportional to $\ln p^{e}$. The smoothness neuron compares the logarithmic sum of factors to threshold $\ln f(x)$ to determine whether $f(x)$ can be completely factored over the neurons that spiked.

Figure 2 depicts neuron activity of the top and bottom layers over time. Activity from tonic neurons $3_{0}$ and $3_{3}^{2}$ is suppressed by neuron $3_{3}^{3}$ at time $t=3$, which ensures only $\ln 3^{3}$ is integrated by the smoothness neuron. A similar situation occurs at time $t=5$. The smoothness neuron spikes at times $\{3,4,5,6\}$ to indicate that polynomial values $\{-27,-10,9,30\}$ are detected as smooth ${ }^{3}$

\footnotetext{
${ }^{3}$ The -1 is treated as an additional factor and is easily accounted for during the linear algebra stage [1]
} 


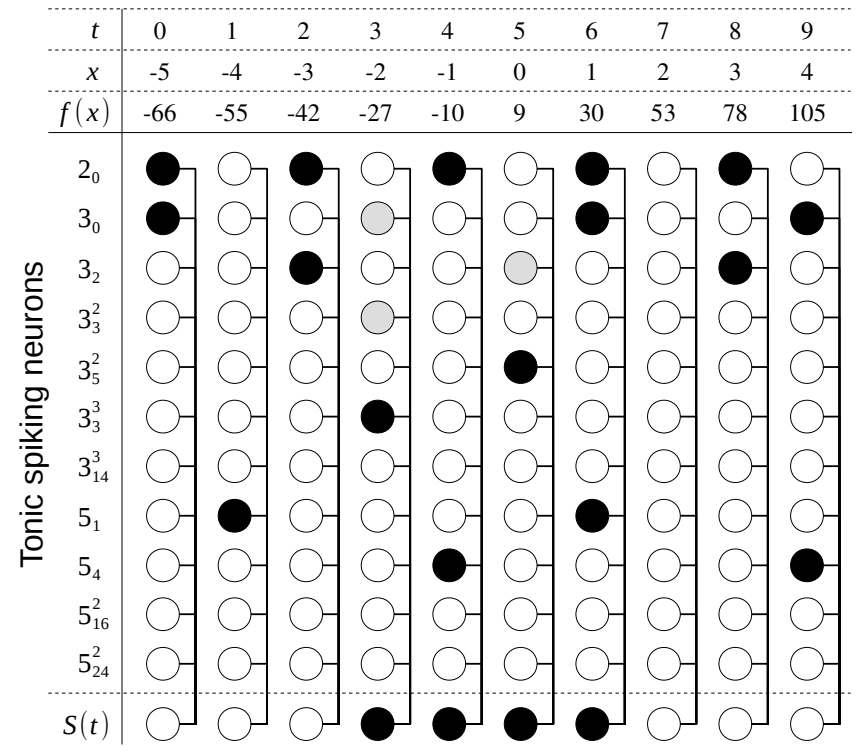

Fig. 2: Neuromorphic sieve example. The smoothness neuron $S$ spikes when smooth values are detected. Active neurons on each time step are shown in black and tonic neurons suppressed by higher prime powers are shown in gray.

\section{TRUENoRTh IMPLEMENTATION}

The TrueNorth architecture employs a digital LIF neuron model given by

$$
V(t)=V(t-1)+\sum_{i} A_{i}(t-1) w_{i}+\lambda
$$

where $V(t)$ is the membrane potential at time $t, A_{i}(t)$ and $w_{i}$ are the $i$ th spiking neuron input and synaptic weight, respectively, and $\lambda$ is the leak [3]. The neuron spikes when the membrane potential crosses a threshold, $V(t) \geq \alpha$, after which it resets to a specified reset membrane potential, $R$. Each neuron can also be configured with an initial membrane potential, $V_{0}$. This behavior is achieved using a subset of the available parameters on the TrueNorth architecture [3].

For each polynomial root $r$ of each prime power $p^{e}$ (including factor base primes, i.e., $e=1$ ), a tonic spiking neuron is configured with period $p^{e}$ and phase $r$, spiking only when $p^{e} \mid f\left(t+x_{\min }\right)$. This is accomplished by setting $\alpha=p^{e}, V_{0}=-\left(r+1-x_{\min }\right) \bmod p^{e}$, and $\lambda=1$. Tonic spiking neurons are configured for prime powers up to $2^{18}$, the maximum period that can be achieved by a single TrueNorth neuron with non-negative membrane potential ( $\alpha$ is an unsigned 18-bit integer). Tonic neurons are connected to the middle layer (factor neurons) through excitatory and inhibitory synapses with weights to invoke or suppress a spike.

TrueNorth implements low-precision synaptic weights through shared axon types. Each neuron can receive inputs from up to 256 axons, and each axon can be assigned one of four types. For each neuron, axons of the same type share a 9bit signed weight. Thus, there can be at most 4 unique weights to any single neuron, and all 256 neurons on the same core must share the same permutation of axon types. This constraint requires the smoothness neuron weights to be quantized.

Four different weight quantization strategies are evaluated: regress fits a single variable regression tree, i.e., step function, with 4 leaf nodes, to the $\log$ factors; inverse fits a similar regression tree using a mean squared error objective function with factors weighted by their log inverse. This forces quantized weights of smaller, frequent factors to be more accurate than large factors; uniform assigns each factor a weight of 1 , thus the smoothness neuron simply counts the number of factors that divide any sieve value; integer assigns each factor a weight equal to the log factor rounded to the nearest integer.

The four quantization strategies are summarized in Figure $3 \mathrm{a}$ Using only integer arithmetic, the integer strategy is optimal as it most closely approximates the log function. The uniform strategy is a worst case in which only binary (0 or 1) weights are available. Note that only the regression, inverse, and uniform strategies, which have at most 4 unique weights, are able to run on TrueNorth. The integer strategy exceeds the limit of 4 unique weights to any single neuron, thus is not compatible with the architecture.

A single neuron $S$ performs the smoothness test by integrating the postsynaptic spikes of the weighted factor neurons. The smoothness neuron is stateless and spikes only when tonic spiking neurons with sufficient postsynaptic potential have spiked on each time step. The stateless behavior of the smoothness neuron is achieved by setting $\alpha=0, R=0$, membrane potential floor to 0 , reset behavior $\kappa=1$, and $\lambda=\tau$, where $\tau$ is a smoothness threshold. Postsynaptic spiking behavior is given by

$$
S(t+1)= \begin{cases}1 & \text { if } \sum A_{i}(t) w_{i} \geq \tau \\ 0 & \text { otherwise }\end{cases}
$$

where $w_{i}$ and $A_{i}(t)$ are the weight and postsynaptic spiking activity of the $i$ th factor neuron, respectively. TrueNorth is a pipelined architecture, and the postsynaptic spikes from the tonic spiking neurons at time $t$ are received by the smoothness neuron at time $t+2$. As a result, the smoothness neuron spikes when $f\left(t+x_{\min }-2\right)$ is likely smooth.

\section{RESULTS}

We modified msieve $\left.\right|^{4}$ to use the IBM Neurosynaptic System (NS1e) [3] for the sieving stage of integer factorization. The NS1e is a single-chip neuromorphic system with 4096 cores and 256 LIF neurons per core, able to simulate a million spiking neurons while consuming under 100 milliwatts at a normal operating frequency of $1 \mathrm{KHz}$. msieve is a highly optimized publicly available implementation of the multiple polynomial quadratic sieve (MPQS, a variant of the quadratic sieve) and NFS factoring algorithms. The core sieving procedure is optimized to minimize RAM access and arithmetic operations. Sieving intervals are broken up into blocks that fit into $\mathrm{L} 1$ processor cache. The host CPU used in this work is a $2.6 \mathrm{GHz}$ Intel Sandy bridge, which has an L1 cache size of

\footnotetext{
${ }^{4}$ msieve version 1.52 , available at https://sourceforge.net/projects/msieve/
} 
64KB. Thus, a sieving interval of length $M=2^{17}$ would be broken up into two blocks that each fit into L1 cache.

Results are obtained for $n$ ranging from 32 to 64 bits in increments of 2, with 100 randomly-chosen $p$ and $q$ of equal magnitude in each setting for a total of 1700 integer factorizations. This range was chosen to limit the number of connections to the smoothness neuron to below 256, as topological constraints are not addressed in this work. For each $n, B$ is set to $\exp \left(\frac{1}{2} \sqrt{\ln n \ln \ln n}\right)$. The factor base size $b$ ranges from 18 for 32-bit $n$ to 119 for 64-bit $n$. Including prime powers, this requires 93 tonic spiking neurons for 32 bit $n$ and 429 tonic spiking neurons for 64-bit $n$, having 47 and 215 connections to the smoothness neuron, respectively. The sieving interval $M$ is set to $2^{17}$, large enough to detect $b+1 B$-smooth numbers in all but 5 cases in which a sieving interval of length $2^{18}$ is used.

The factor base primes for each $n$ are determined by msieve and then used to construct the neuromorphic sieve on the TrueNorth architecture, as described in Section IV] Polynomial roots of the prime factors are calculated efficiently by the Tonelli-Shanks algorithm, and roots of prime powers are obtained through Hensel lifting. The resulting network is deployed to the NS1e, which runs for $M+2$ time steps. On each time step, if a smooth value is detected, a spike is generated and transmitted to the host which then checks the corresponding polynomial value for smoothness.

Figure 3 summarizes the results. ROC curves obtained using each quantization strategy for 64-bit $n$ are shown in Figure $3 \mathrm{~b}$ The inverse strategy, which has a $0.525 \pm 0.215 \%$ equal error rate (EER), performs nearly as well as the optimal integer strategy having a $0.318 \pm 0.220 \%$ EER. Results for the integer strategy are obtained by integrating the bottom layer of the neuromorphic sieve off of the TrueNorth chip.

Figure $3 \mathrm{c}$ shows the number of clock cycles per sieve value as a function of $\log _{2} n$ (bits). This metric remains constant for the neuromorphic sieve, which performs a test for smoothness within one clock cycle using any quantization strategy. CPU cycles were measured using differences of the RDTSC instruction around sieving routines on a single dedicated core of the host CPU.

Figure 3d shows the FPR of each quantization strategy at the point on the ROC curve where true positive rate (TPR) equals the CPU TPR. For all $n$, and given the same level of sensitivity, the neuromorphic sieve with binary weights (uniform strategy) demonstrates equal or higher specificity, i.e., lower FPR, than the CPU-based sieve and significantly lower FPR using quaternary weights (regress and inverse strategies). The higher-precision integer weights performed marginally better than quaternary weights.

\section{CONCLUSION}

This work highlights the ability of a neuromorphic system to perform computations other than machine learning. A $O(1)$ test for detecting smooth numbers with high probability is achieved, and in some cases is significantly more accurate than a CPU-based implementation which performs the same

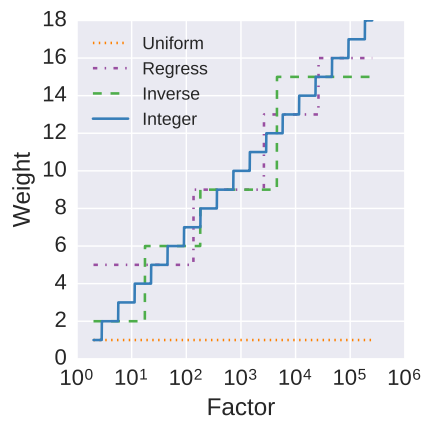

(a) Weight quantization.

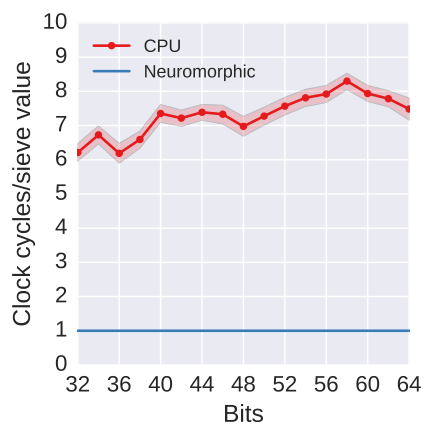

(c) Clock cycles/sieve value vs $n$ bits

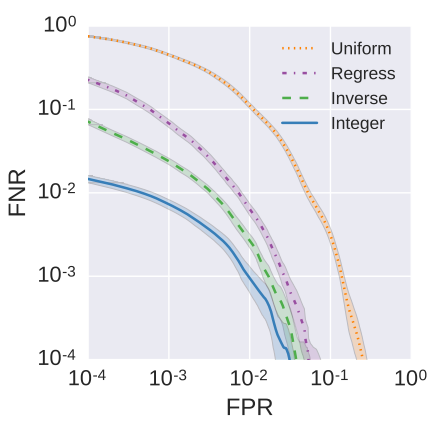

(b) 64-bit $n$ ROC curves.

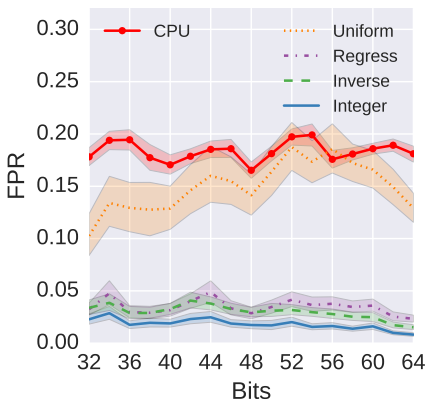

(d) FPR given CPU TPR vs $n$ bits.
Fig. 3: Experimental results. Bands show 95\% confidence intervals obtained over 100 different $n$ of equal magnitude.

operation in $O(\ln \ln B)$ amortized time. Despite this, the NS1e has a normal operating frequency of $1 \mathrm{KHz}$ and wall clock time is only asymptotically lower than that of the CPU. Future high-frequency neuromorphic architectures may be capable of sieving large intervals in a much shorter amount of time. How such topological and precision constraints determine the accuracy of these architectures is an item for future work.

It is also worth noting that since the NS1e is a digital architecture, at the hardware level it does not achieve constant time synaptic integration. The ability to perform constant time addition is promised only by analog architectures that exploit the underlying physics of the device to compute, for example by integrating electrical potential (memristive architecture) or light intensity (photonic architecture).

\section{REFERENCES}

[1] Richard Crandall and Carl Pomerance. Prime numbers: a computational perspective, volume 182. Springer, 2006.

[2] Andrew Granville. Smooth numbers: computational number theory and beyond. Algorithmic number theory: lattices, number fields, curves and cryptography, 44:267-323, 2008.

[3] Paul A Merolla et al. A million spiking-neuron integrated circuit with a scalable communication network and interface. Science, 345(6197):668673, 2014.

[4] Peter L Montgomery. A block lanczos algorithm for finding dependencies over gf (2). In Advances in cryptology-EUROCRYPT'95, pages 106-120. Springer, 1995.

[5] Carl Pomerance. The quadratic sieve factoring algorithm. In Advances in cryptology, pages 169-182. Springer, 1984.

[6] Carl Pomerance. The role of smooth numbers in number theoretic algorithms. In Proc. Internat. Congr. Math., Zürich, Switzerland, volume 1, pages 411-422, 1994. 